\title{
A New Cold Plasma Jet: Performance Evaluation of Cold Plasma, Hybrid Plasma and Argon Plasma Coagulation
}

\author{
Lawan Ly ${ }^{1}$, Sterlyn Jones ${ }^{1}$, Alexey Shashurin ${ }^{1,2}$, Taisen Zhuang ${ }^{3}{ }^{(0)}$, Warren Rowe III ${ }^{1}$ (D), \\ Xiaoqian Cheng $\left.{ }^{1}{ }^{(}\right)$, Shruti Wigh ${ }^{3}$, Tammey Naab ${ }^{4}$, Michael Keidar ${ }^{1,5}$ and Jerome Canady ${ }^{1,5, *}$ \\ 1 Jerome Canady Research Institute for Advanced Biological and Technological Sciences, Takoma Park, \\ MD 20912, USA; llawan@usmedinnov.com (L.L.); sterjones78@aol.com (S.J.); ashashur@purdue.edu (A.S.); \\ drwrowe@usmedinnov.com (W.R.); xcheng@usmedinnov.com (X.C.); keidar@email.gwu.edu (M.K.) \\ 2 Department of Aerospace Engineering, Purdue University, West Lafayette, IN 47907, USA \\ 3 Plasma Medicine Life Sciences, Takoma Park, MD 20912, USA; tzhuang@usmedinnov.com (T.Z.); \\ swigh@usmedinnov.com (S.W.) \\ 4 Department of Pathology, Howard University Hospital, Washington, DC 20059, USA; tjnaab@gmail.com \\ 5 Department of Mechanical and Aerospace Engineering, The George Washington University, Washington, \\ DC 20052, USA \\ * Correspondence: drjcanady@usmedinnov.com; Tel.: +1-301-270-0147
}

Received: 2 August 2018; Accepted: 7 September 2018; Published: 11 September 2018

\begin{abstract}
The use of plasma energy has expanded in surgery and medicine. Tumor resection in surgery and endoscopy has incorporated the use of a plasma scalpel or catheter for over four decades. A new plasma energy has expanded the tools in surgery: Cold Atmospheric Plasma (CAP). A cold plasma generator and handpiece are required to deliver the CAP energy. The authors evaluated a new Cold Plasma Jet System. The Cold Plasma Jet System consists of a USMI Cold Plasma Conversion Unit, Canady Helios Cold Plasma ${ }^{\circledR}$ Scalpel, and the Canady Plasma ${ }^{\circledR}$ Scalpel in Hybrid and Argon Plasma Coagulation (APC) modes. This plasma surgical system is designed to remove the target tumor with minimal blood loss and subsequently spray the local area with cold plasma. In this study, various operational parameters of the Canady Plasma ${ }^{\circledR}$ Scalpels were tested on ex vivo normal porcine liver tissue. These conditions included various gas flow rates $(1.0,3.0,5.0 \mathrm{~L} / \mathrm{min})$, powers $(20,40$, $60 \mathrm{P})$, and treatment durations $(30,60,90,120 \mathrm{~s})$ with argon and helium gases. Plasma length, tissue temperature changes, and depth and eschar injury magnitude measurements resulting from treatment were taken into consideration in the comparison of the scalpels. The authors report that a new cold plasma jet technology does not produce any thermal damage to normal tissue.
\end{abstract}

Keywords: cold atmospheric plasma; cold plasma device; Hybrid plasma; argon plasma coagulation

\section{Introduction}

Electrosurgery is the use of high frequency radiofrequency (RF) alternating current (AC) for cutting and coagulation of tissue. Investigators in Europe and the United States started to explore the effect of AC on tissue in the late nineteenth century. In 1893, a French inventor and physicist, Arsene d'Arsonval was the first to report the clinical effect of AC on tissue [1]. Many Europeans and Americans contributed to the development of the AC electrosurgical generator but the invention by Dr. William T. Bovie [2] stands out the most. Bovie's contributions in electrosurgery enhanced after collaborating with Dr. Harvey Cushing, Surgeon in Chief at Peter Bent Brigham, who used Bovie's electrosurgical generator for a brain tumor because of excessive bleeding [3]. 
Plasma technology in surgery has advanced since the first introduction by Morrison 44 years ago [4]. In 1990, Canady [5] was the first to describe the delivery of argon plasma coagulation via a flexible endoscope. These new methods allowed the surgeon and endoscopist to combine standard monopolar electrocautery with a plasma gas for the coagulation of tissue. In 2010, Canady et al. [6] developed a new mode of plasma- "Hybrid Plasma". Hybrid plasma combines monopolar electrical charge with a plasma gas which creates a plasma beam that can simultaneously cut and coagulate biological tissue.

After the introduction of Canady's Hybrid plasma, we continued to develop several electrosurgical systems. In our previous studies, we tested an older generation of our Hybrid electrosurgical system (SS-200E/Argon 2) using an argon flow rate of $3 \mathrm{~L} / \mathrm{min}$ and powers of 40,60, and $80 \mathrm{~W}[7,8]$. Pure cutting [7] and pure coagulation [8] was successfully demonstrated on various animal biological samples. In another study, we compared our SS-200E/Argon 2 and SS-501MCa/Argon 4 electrosurgical systems in terms of the plasma properties and injury characteristics produced [9]. Conventional coagulation, argon coagulation, conventional cut, and argon cut modes were explored with treatment times totaling $5 \mathrm{~s}$ with various flow rates and powers. Both electrosurgical systems operating under argon cut mode and gentle parameters exhibited injury sizes suitable for surgical procedures.

We have recently developed a series of electrosurgical systems compromised of the Canady Helios Plasma ${ }^{\circledR}$ Scalpel with Cold Atmospheric Plasma (CAP) mode (operating with the SS-601MCa generator and USMI Cold Plasma Conversion Unit) [10] and the Canady Plasma ${ }^{\circledR}$ Scalpel, which is capable of Hybrid and Argon Plasma Coagulation (APC) modes (operating on the USMI SS-601MCa/Argon 4 generator). The Canady Plasma ${ }^{\circledR}$ Scalpel in Hybrid mode, capable of simultaneous cut and coagulation, has the potential to surgically remove the target tumor while coagulating the surrounding tissue. In addition, the Canady Plasma ${ }^{\circledR}$ Scalpel in APC mode can aid in the procedure by providing pure coagulation. The coagulation capabilities of Hybrid and APC modes could minimize blood loss during the surgical procedure by sealing off blood vessels, which in turn may reduce post-operative complications that arise from hemorrhaging. After the tumor is removed using the Canady Plasma ${ }^{\circledR}$ Scalpel in Hybrid and APC modes, the Canady Helios Plasma ${ }^{\circledR}$ Scalpel can be used to spray the surgical margins with CAP. The treatment with CAP will eliminate undetected cancer cells, thus preventing tumor recurrence.

Recently, CAP has been gaining more interest as a potential cancer treatment due to promising research results. CAP is known to selectively ablate cancer cells in vitro and reduce tumor size in vivo [11,12]. The reactive oxygen and nitrogen species (RONS) generated by CAP has been recognized as a possible factor in selective eradication [12-14]. It has been observed that RONS induce apoptosis in cancer cells through an oxidative DNA damage cascade [15] and downregulation of oxidative stress-related genes [16]. To this end, selective treatment of surgical margins allowing elimination of cancerous areas has tremendous advantage; this could be provided by the Canady Helios Plasma ${ }^{\circledR}$ Scalpel.

In our recent study, we have shown that the spectrum of CAP, generated by the USMI Cold Plasma Conversion Unit and Canady Helios Cold Plasma Scalpel, mainly consists of $\mathrm{N}_{2}, \mathrm{~N}_{2}{ }^{+}, \mathrm{He}$, and O [17]. We have also demonstrated that the CAP jet significantly reduced cell viability in a dose-dependent manner in various cell lines such as 769-P (renal adenocarcinoma), HCT-116 (colorectal carcinoma), SK-OV-3 (ovarian adenocarcinoma), BxPC-3 (pancreatic adenocarcinoma), OE33 (oesophageal adenocarcinoma), and MDA-MB-231 (breast adenocarcinoma) [17,18]. With various CAP dosages, an $80-99 \%$ reduction in viability of these cancer cells lines was achieved $48 \mathrm{~h}$ after treatment [17]. However, the safety of this new device must be taken into consideration.

In this study, we evaluated the extent of the thermal injuries produced by the Canady Plasma ${ }^{\circledR}$ Scalpels in combination with our new generation of generators (SS-601MCa generator/USMI Cold Plasma Conversion Unit and USMI SS-601MCa/Argon 4 generator) (US Medical Innovations, Takoma Park, MD, USA). Various parameters of the Canady Plasma ${ }^{\circledR}$ Scalpels were tested to compare the severity of injuries produced by CAP, Hybrid, and APC modes. 


\section{Materials and Methods}

\subsection{Canady Plasma ${ }^{\circledR}$ Scalpels}

All experiments were performed at the Jerome Canady Research Institute for Advanced Biological and Technological Sciences, (JCRI-ABTS), Takoma Park, MD, USA. All experiments were conducted using the Canady Helios Cold Plasma ${ }^{\circledR}$ Scalpel and the Canady Plasma Scalpel in Hybrid and APC Mode, SS-601/Cold Plasma Converter (US Medical Innovations, LLC., Takoma Park, MD, USA), pictured in Figure 1. Each Canady Plasma ${ }^{\circledR}$ Scalpel has a channel and an electrode with an opening at the end. An inert gas flows through the channel to the electrode where a high-frequency energy is applied to the electrode causing the gas to ionize as it exits the port. Helium gas is used for the Canady Helios Plasma ${ }^{\circledR}$ Scalpel and argon gas for the Canady Plasma ${ }^{\circledR}$ Scalpel in Hybrid and APC modes.

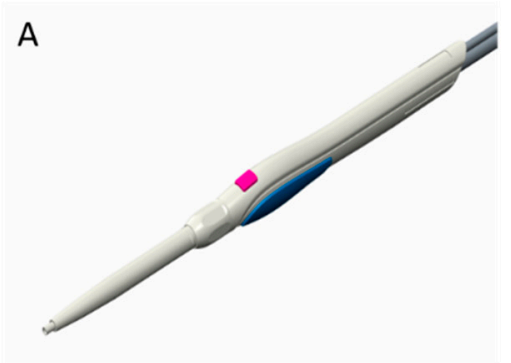

B

Figure 1. (A) Canady Helios Cold Plasma ${ }^{\circledR}$ Scalpel and (B) Canady Plasma ${ }^{\circledR}$ Scalpel with Hybrid and Argon Plasma Coagulation (APC) Modes.

\subsubsection{CAP Mode}

The Canady Helios Plasma ${ }^{\circledR}$ Scalpel utilizes the USMI SS-601MCa generator with a USMI Cold Plasma Conversion Unit to produce CAP. The conversion unit up-converts voltage $(1.5-50 \mathrm{kV})$, down-converts frequency $(<300 \mathrm{kHz})$, and down-converts power $(<30 \mathrm{~W})$.

\subsubsection{APC Mode}

The Candy Plasma ${ }^{\circledR}$ Scalpel in APC mode operates on the Hybrid electrosurgical system which includes the USMI SS-601MCa/Argon 4. For pure coagulation, only the Argon 4 generator is required. Coagulation requires a high voltage waveform with a low duty cycle. The surges in voltage power, referred to as spikes, are necessary for coagulation. When the surrounding tissue is heated during the spike and cooled between spikes, tissue coagulation is achieved.

\subsubsection{Hybrid Mode}

Hybrid mode relies on the USMI SS-601MCa/Argon 4 for simultaneous cutting and coagulation. The Argon 4 coagulator provides the argon gas. To first initiate a cutting action, a sufficient amount of power is required for the formation of a steam layer between the electrode and the tissue. The steam layer subsequently allows for the formation of a plasma composed of highly ionized argon and water. A radiofrequency arc, characterized by high power density, is then developed in the plasma. Upon contact with the tissue, the arc causes immediate disruption in tissue structure. A low voltage waveform with high duty cycle is required to maintain a constant succession of arcs to produce a cut in the tissue.

To achieve simultaneous cutting and coagulation under Hybrid mode, the continuous sinusoidal voltage used for cutting is periodically interrupted. During interruptions, the ionized argon particles in the plasma disperse allowing the electrode to temporarily make direct contact with the tissue. This interaction causes coagulation. 


\subsection{Preparation and Treatment Conditions of Liver Tissue Samples}

All experiments were conducted on ex vivo normal porcine liver tissue. Tissue was divided into $1.5 \mathrm{~cm} \times 1.5 \mathrm{~cm}$ samples and kept in a $15^{\circ} \mathrm{C}$ environment prior to treatment. Samples were treated with gas flow rates of 1.0,3.0, and $5.0 \mathrm{~L} / \mathrm{min}$. Power settings were tested at 20, 40, and $60 \mathrm{P}$ which corresponded to 5, 8, $11 \mathrm{~W}$ when using the USMI Cold Plasma ${ }^{\circledR}$ Conversion Unit and 20, 40, $60 \mathrm{~W}$ on the Argon 4/SS-601MCa. Exposure times to the plasma were set to 30, 60, 90, and $120 \mathrm{~s}$. Equipment set up is displayed in Figure 2.

A

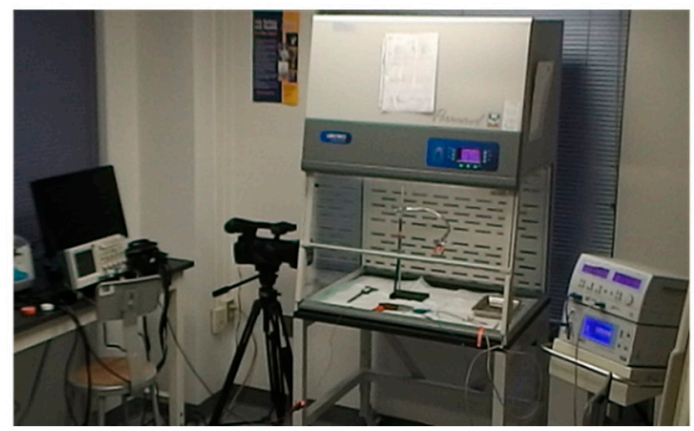

B

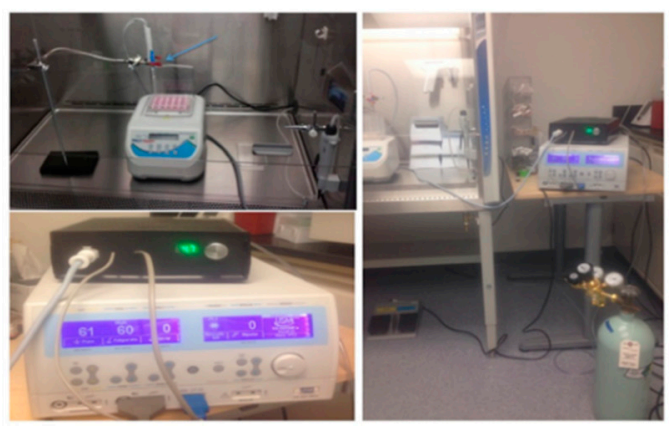

Figure 2. Overall equipment setup with the USMI SS-601MCa generator/USMI Cold Plasma Conversion Unit in (A) and the USMI SS-601MCa/USMI Argon 4 generator in (B).

Plasma lengths of the Canady Plasma ${ }^{\circledR}$ Scalpels were measured and defined as the maximum length of the plasma beam at which the discharge was sustained. The treatments were video recorded by digital camera (Panasonic AG, Panasonic, Kadoma, Osaka Prefecture, Japan).

Tissue temperature was recorded with infrared temperature sensor FLIR (E Series) before and after treatment. The accuracy of the temperature measurements was less than $12{ }^{\circ} \mathrm{C}$.

The Escher diameter of the injury was measured with a 6-inch digital caliper post-treatment. The accuracy of the diameter measurements was less than $0.05 \mathrm{~mm}$.

The depth of injury was determined by examining stained slides of treated tissue samples. Preparation of the slides began immediately after plasma treatment; samples were fixed in $4 \%$ buffered formalin for $5 \mathrm{~h}$, paraffin embedded, and stained for hematoxylin and eosin. Preparation of the pathological slides was performed at the Histopathology Laboratories: Howard University Hospital, Washington, DC, USA and JCRI-ABTS, Takoma Park, MD, USA. The slides were studied under the Zeiss Primovert phase contrast microscope with Zeiss ZEN SP1/SP2 blue edition software (Zeiss, Oberkochen, Germany). Data for plasma length, tissue temperature change, eschar width, and depth of injury for all treatment conditions has been included as Supplementary Materials.

\subsection{Statistics}

All treatment conditions were repeated 3 times with the exception for plasma length on Hybrid mode (which were negligible and repeated once) and for depth of injury on Hybrid and APC modes (which were repeated 1-3 times). A non-parametric student two-tailed, unpaired $t$ test was performed for all samples $n=3$. ${ }^{*} p$-value $\leq 0.05$ was considered significant and a mean difference at $95 \%$ confidence level was used with SPSS software (IBM, New York, NY, USA). 


\section{Results}

\subsection{Plasma Length Measurements}

Figure 3 displays plasma discharge produced by each mode. Hybrid plasma is initiated upon contact as seen in Figure 3A. And unlike Hybrid mode, CAP (Figure 3B) and APC (Figure 3C) modes can be held a distance away from the tissue thus do not require contact for plasma discharge.

A

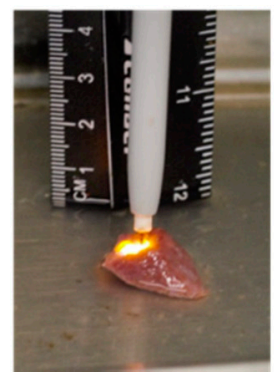

B

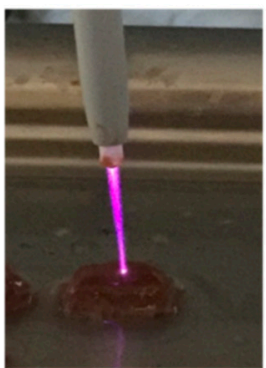

C

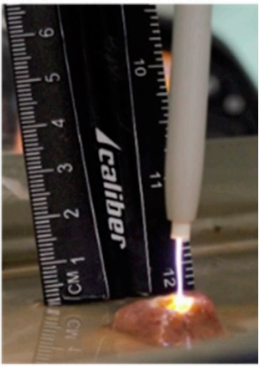

Figure 3. Plasma discharge in (A) Hybrid; (B) CAP; and (C) APC modes.

Figure 4 focuses on the solely on the CAP beam. The discharge elongates with flow rate as the beam lengths increase in ascending order of $1 \mathrm{~L} / \mathrm{min}$ (Figure $4 \mathrm{~A}$ ), $3 \mathrm{~L} / \mathrm{min}$ (Figure $4 \mathrm{~B}$ ), and $5 \mathrm{~L} / \mathrm{min}$ (Figure 4C). Light intensity emitted from the discharge strengthens with power, shown from left to right in all rows of Figure 4.

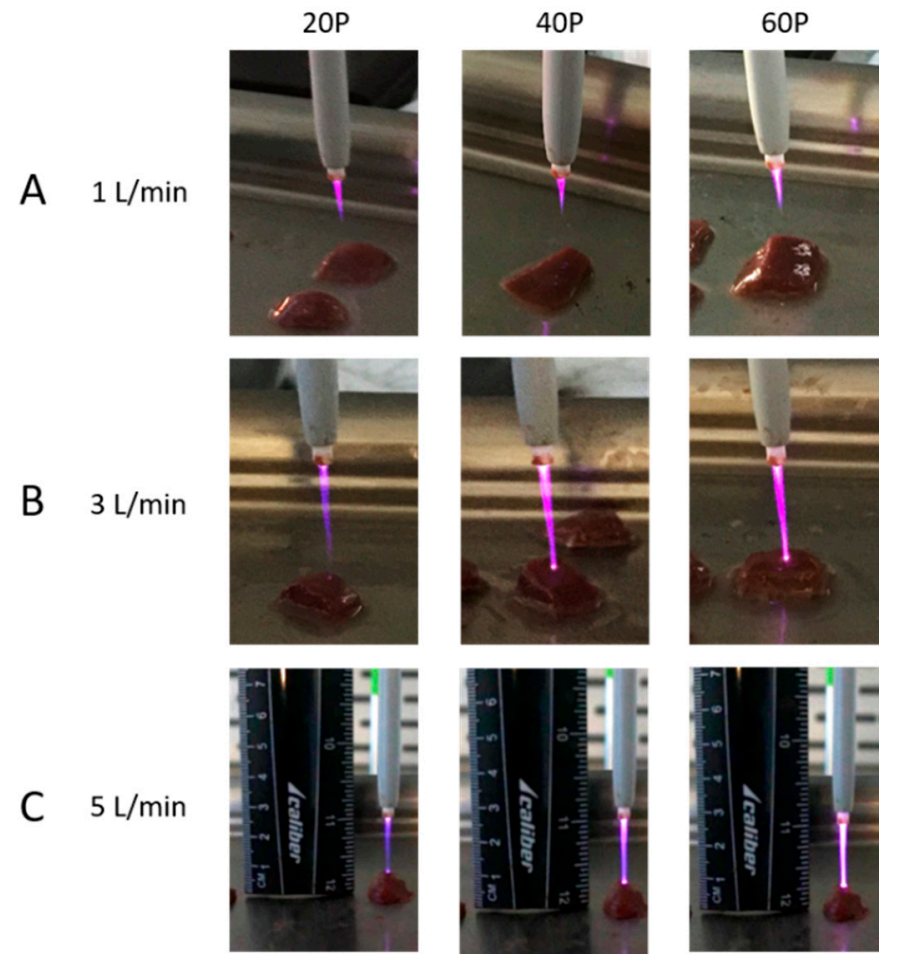

Figure 4. All treatments with the Canady Helios Cold Plasma ${ }^{\circledR}$ Scalpel were maintained at a 2-cm distance from the tissue sample. The CAP beam transformed in length and light intensity under 1 $\mathrm{L} / \min (\mathbf{A}) ; 3 \mathrm{~L} / \mathrm{min}(\mathbf{B})$; and $5 \mathrm{~L} / \mathrm{min}(\mathbf{C})$ flow rates and 20, 40, $60 \mathrm{P}$.

The maximum plasma length generated by each electrosurgical scalpel using various gas flow rates, powers, and treatment durations is shown in Figure 5. The Canady Helios Cold Plasma ${ }^{\circledR}$ Scalpel 
was constantly held $2 \mathrm{~cm}$ away from the tissue surface, hence, the plasma length for cold plasma mode was consistently recorded at $2 \mathrm{~cm}$. Plasma length for Hybrid mode was negligible at 0.025 to $0.45 \mathrm{~mm}$ for all conditions. Argon mode demonstrated the most flexibility in plasma length which ranged from 5-20 mm. In the case of APC, one can see that the plasma column length varies with power applied (Figure 5A-I).

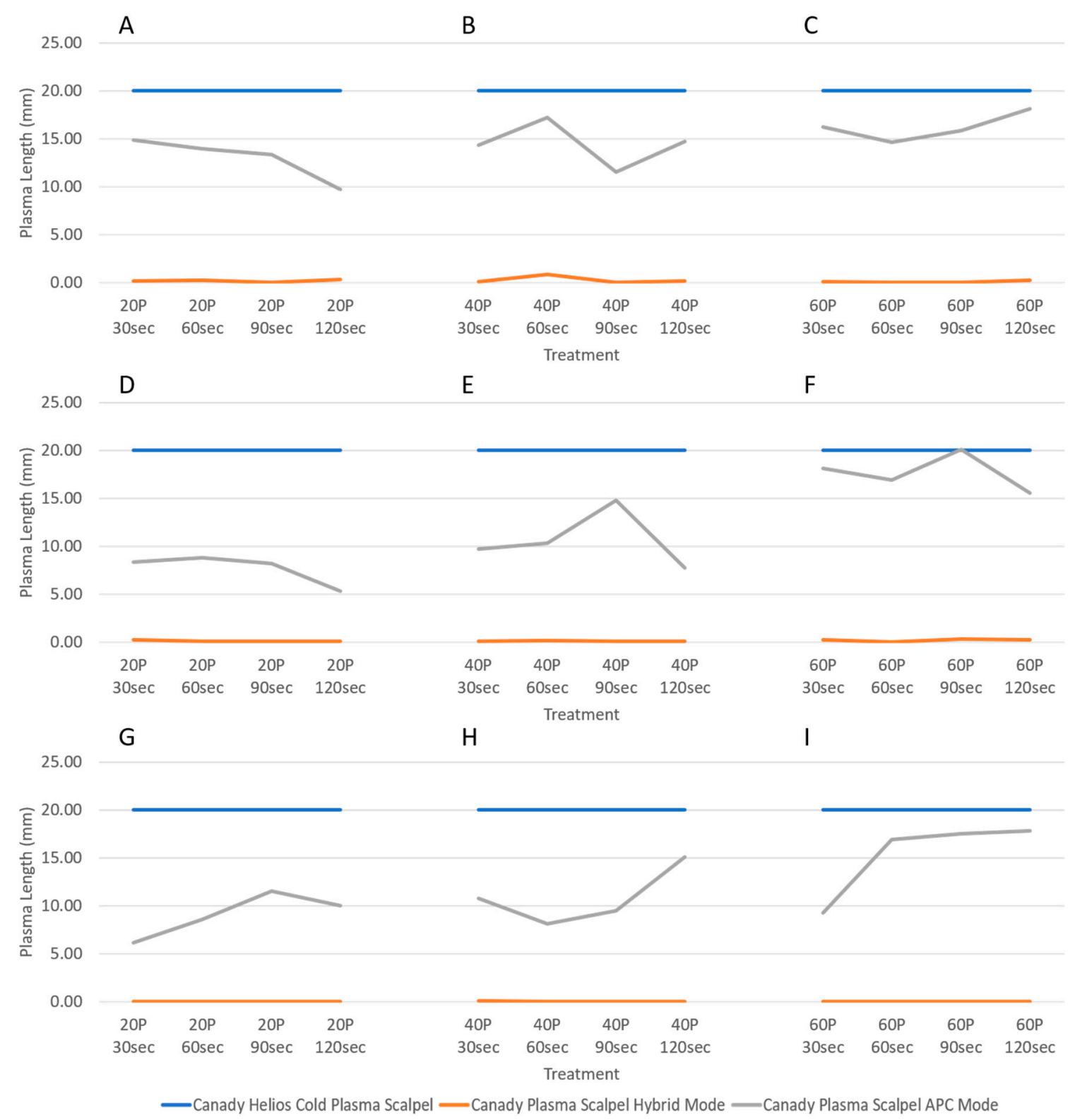

Figure 5. Plasma length of each scalpel was measured during treatments. Treatment conditions were $1 \mathrm{~L} / \mathrm{min}(\mathbf{A}-\mathbf{C}), 3 \mathrm{~L} / \mathrm{min}(\mathbf{D}-\mathbf{F})$, and $5 \mathrm{~L} / \mathrm{min}(\mathbf{G}-\mathbf{I})$ flow rates with different powers and durations.

\subsection{Temperature Change Measurements}

Initial temperatures for all tissue samples were estimated to be $15^{\circ} \mathrm{C}$. Total temperature changes in result of the three modes of plasma treatments are displayed in Figure 6. It was clearly demonstrated that both CAP and Hybrid modes produced significantly lower tissue temperatures than APC modes despite increasing plasma dosages (Figure 6A-F). Argon mode proved to induce the greatest thermal effect as final temperatures ascended to 8 to 10-fold at $5 \mathrm{~L} / \mathrm{min}$ (Figure 6G-I). Under 1 and $3 \mathrm{~L} / \mathrm{min}$, it is difficult to establish a definite relationship between the operational parameters of APC and 
temperature change (Figure 6A-F). However, it was observed that $5 \mathrm{~L} / \mathrm{min}$ yielded higher final tissue temperatures with longer treatment times until a plateau was reached (Figure 6G-I).

A

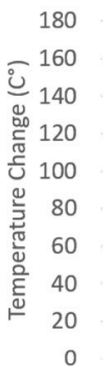

180

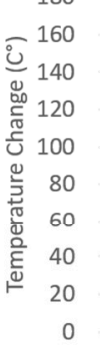

B

C

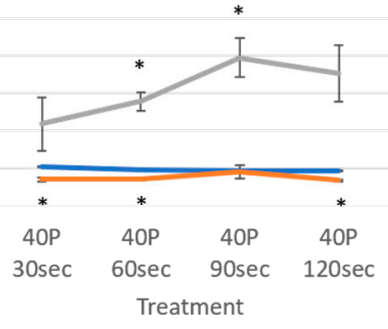

$E$

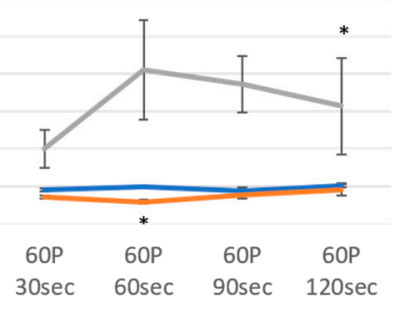

$\mathrm{F}$

—Canady Helios Cold Plasma Scalpel —Canady Plasma Scalpel Hybrid Mode —Canady Plasma Scalpel APC Mode

Figure 6. Total temperature changes produced by each scalpel was measured post-treatment. Treatment conditions were $1 \mathrm{~L} / \mathrm{min}(\mathbf{A}-\mathbf{C}), 3 \mathrm{~L} / \mathrm{min}(\mathbf{D}-\mathbf{F})$, and $5 \mathrm{~L} / \mathrm{min}(\mathbf{G}-\mathbf{I})$ flow rates with different powers and durations.

\subsection{Variability in Injury Magnitude: Eschar Diameter}

Post-treatment tissue morphology in result of Hybrid mode and APC modes using $3.0 \mathrm{~L} / \mathrm{min}$ and $40 \mathrm{P}$ for $90 \mathrm{~s}$ are shown Figure 7 . It is demonstrated that even under the same condition, injury magnitude strongly differs between the two modes. One can observe that the injury produced by Hybrid mode (Figure 7A) is both smaller in depth and eschar diameter and lighter in color than that produced by APC mode (Figure 7B), due to the vast difference in plasma discharge temperature. It can be assumed that dramatic temperature heating leads to greater cell death which is reflected in the greater injury magnitude and darkening of tissue color produced by APC. 
A

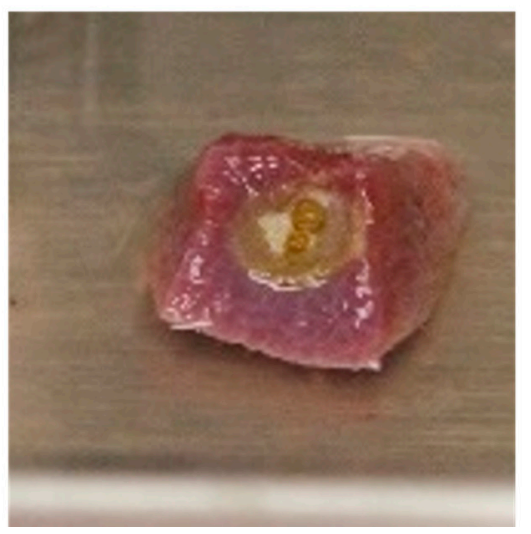

B

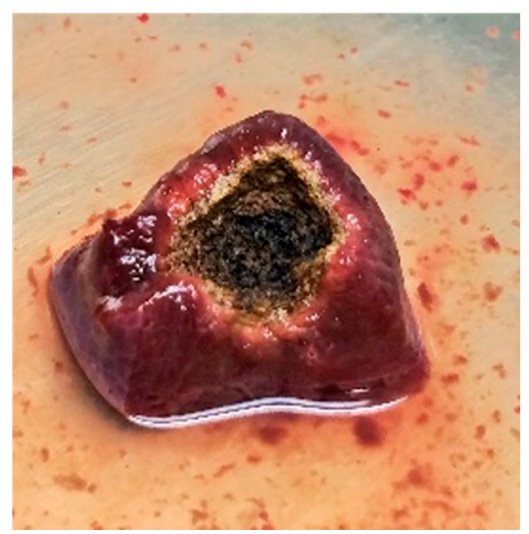

Figure 7. Demonstration of injury magnitude by Hybrid mode (A) and APC (B) mode using $3.0 \mathrm{~L} / \mathrm{min}$ and $40 \mathrm{P}$ for a total of $90 \mathrm{~s}$.

Figure 8 presents samples of the pathology images taken after CAP treatment. It shows no eschar diameter injury produced by CAP treatment with settings of 1 (Figure 8A), 3 (Figure 8B), 5 (Figure $8 \mathrm{C}$ ) L/min at $40 \mathrm{P}$ and $60 \mathrm{~s}$.

A

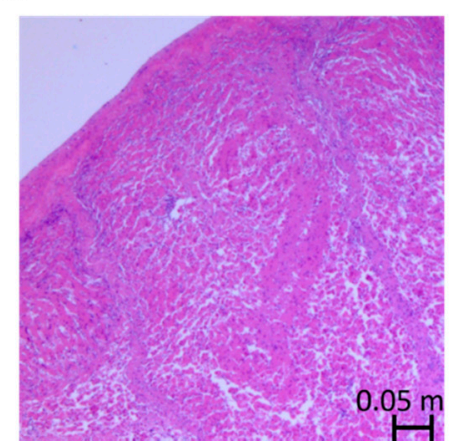

B

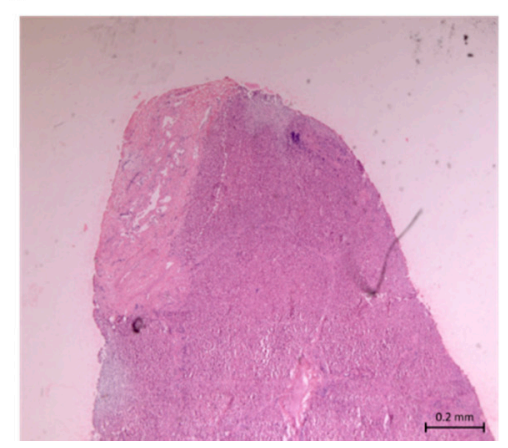

C

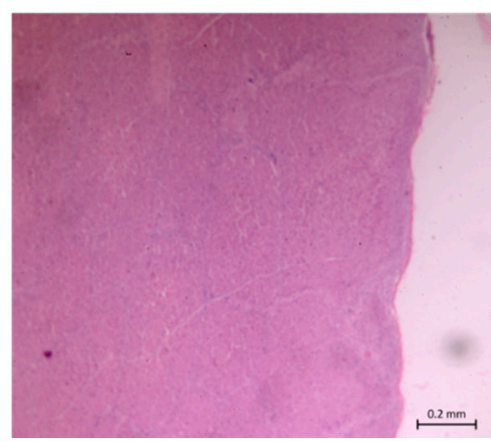

Figure 8. The CAP treatment with $1 \mathrm{~L} / \mathrm{min}(\mathbf{A}) ; 3 \mathrm{~L} / \mathrm{min}(\mathbf{B}) ; 5 \mathrm{~L} / \mathrm{min}(\mathrm{C})$ and $40 \mathrm{P}$ for $60 \mathrm{~s}$ causes no eschar diameter and depth of injury.

Figure 9 presents the average eschar diameter induced by each electrosurgical device. CAP showed no thermal injury as the diameter of injury remained at zero regardless of treatment condition. Injury by Hybrid and APC mode ranged from 1-8 $\mathrm{mm}$ and 8-18 $\mathrm{mm}$ in diameter, respectively. In both Hybrid and APC mode, change in diameter of injury appears to be independent of gas flow rate. Instead, greater injury is observed with an increase in power. While longer treatment durations with Hybrid plasma seems to have no effect on injury diameter, the results reveal that longer exposure to argon plasma is a factor in the increase of injury diameter. 
A

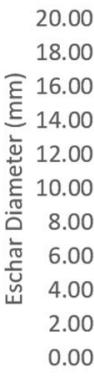

20.00

18.00

E 16.00

है 14.00

屯 12.00

है 10.00

. 8.00

范 6.00

岀 4.00

2.00

0.00

20.00

18.00

छ 16.00

है 14.00

¿ั 12.00

है 10.00

. 8.00

ำ 6.00

岕 4.00

2.00

0.00

D

G
B
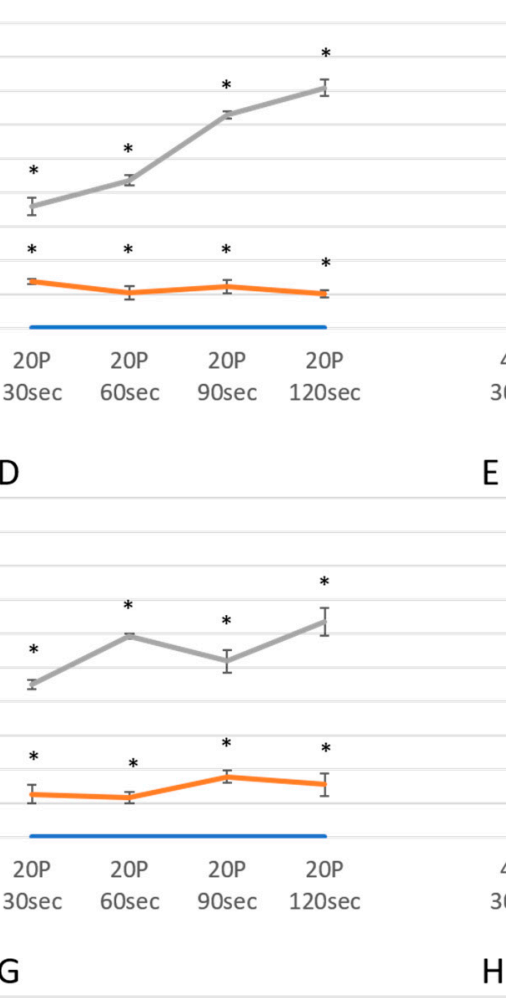

$\mathrm{H}$

E
C
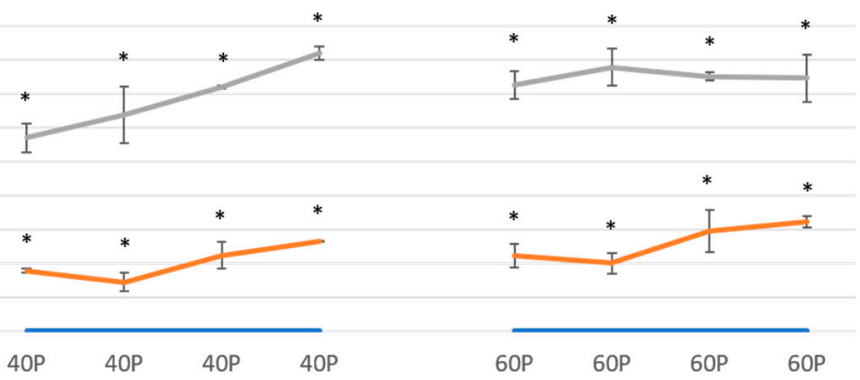

$30 \mathrm{sec} 60 \mathrm{sec} 90 \mathrm{sec} 120 \mathrm{sec}$

Treatment

F
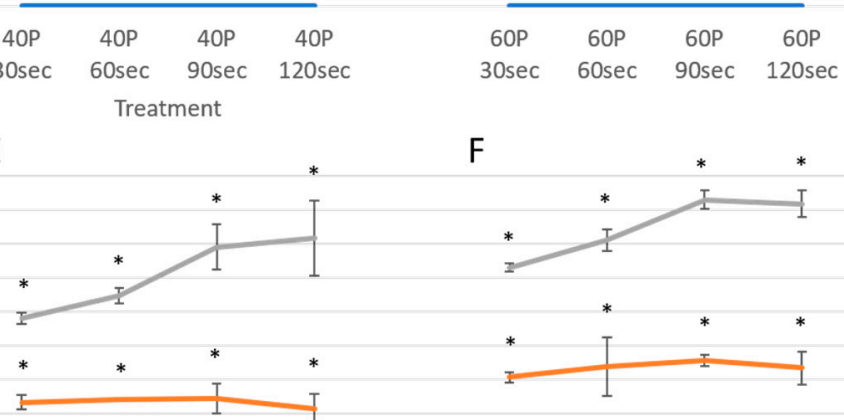

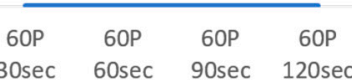

$30 \mathrm{sec} 60 \mathrm{sec} 90 \mathrm{sec} 120 \mathrm{sec} \quad 30 \mathrm{sec} 60 \mathrm{sec} 90 \mathrm{sec} 120 \mathrm{sec}$

Treatment

I

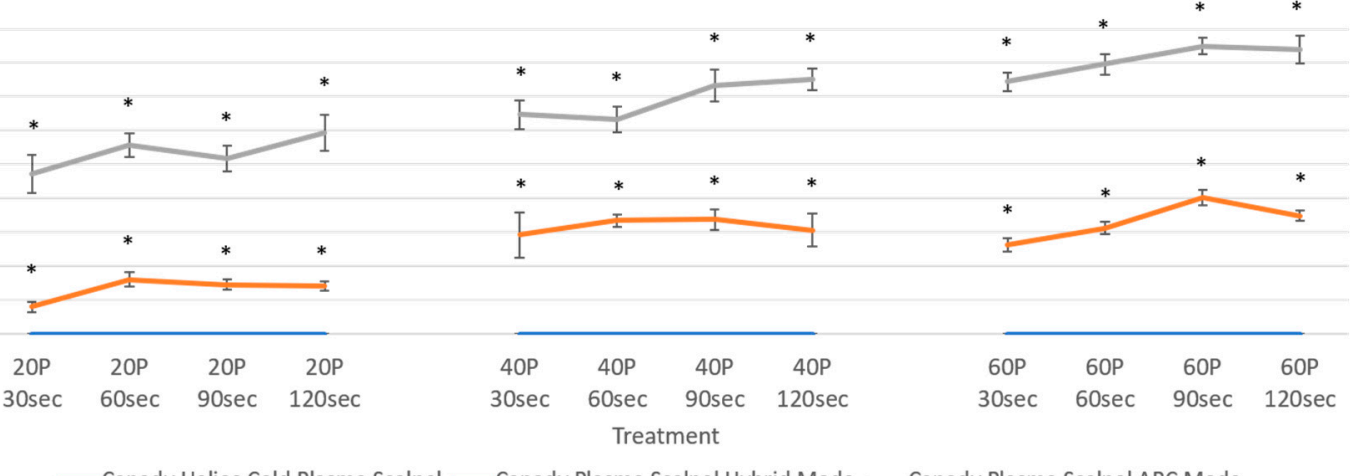

—Canady Helios Cold Plasma Scalpel —Canady Plasma Scalpel Hybrid Mode —Canady Plasma Scalpel APC Mode

Figure 9. Eschar diameter caused by each scalpel was measured post-treatment. Treatment conditions were $1 \mathrm{~L} / \mathrm{min}(\mathbf{A}-\mathbf{C}), 3 \mathrm{~L} / \mathrm{min}(\mathbf{D}-\mathbf{F})$, and $5 \mathrm{~L} / \mathrm{min}(\mathbf{G}-\mathbf{I})$ flow rates with different powers and durations.

\subsection{Variability in Injury Magnitude: Depth of Injury}

Depth of injury under various device parameters are presented in Figure 10. With CAP treatment, depth of injury was $0.0 \mathrm{~mm}$ for all conditions (Figures $8 \mathrm{~A}-\mathrm{C}$ and $10 \mathrm{~A}-\mathrm{I}$ ). The plasmas generated by Hybrid and APC modes led to relatively greater depth of injury at $0.1-0.8 \mathrm{~mm}$ and $0.3-1.5 \mathrm{~mm}$ deep, respectively (Figure 10A-I). While it is difficult to establish a definite relationship between the parameters of Hybrid and depth of injury, it was observed that power increases depth of injury under APC mode. 
A

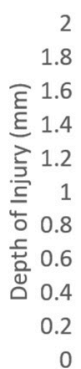

2

1.6

है 1.4

1.2

근 1

0.8
点 0.6

0.4

0

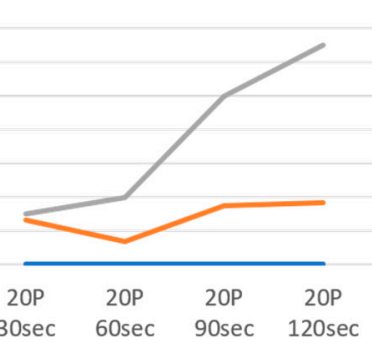

D

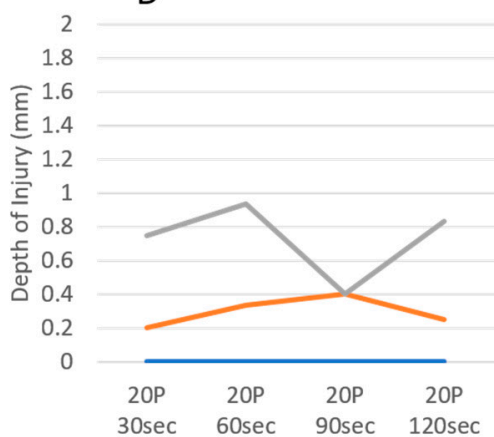

G
B

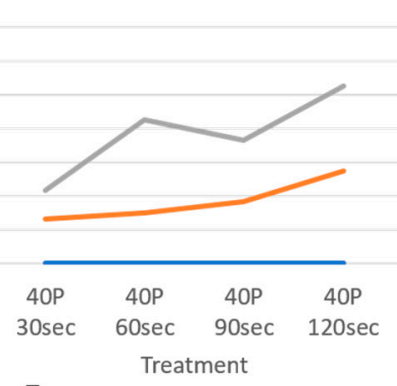

E
C

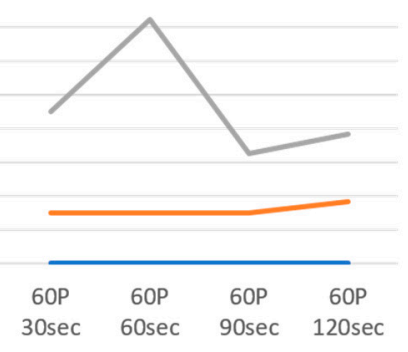

F

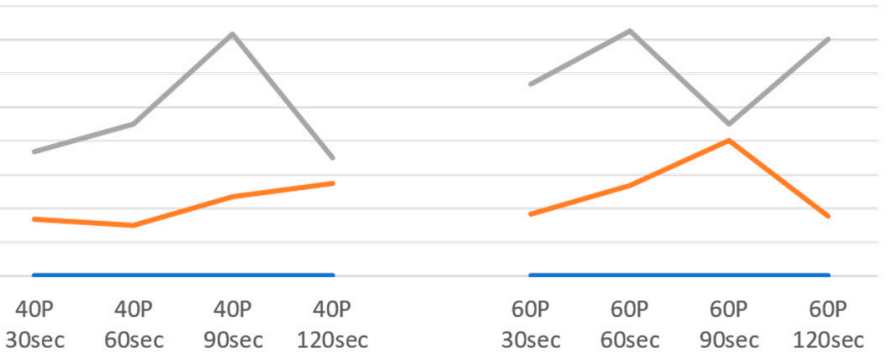

$\mathrm{H}$

I

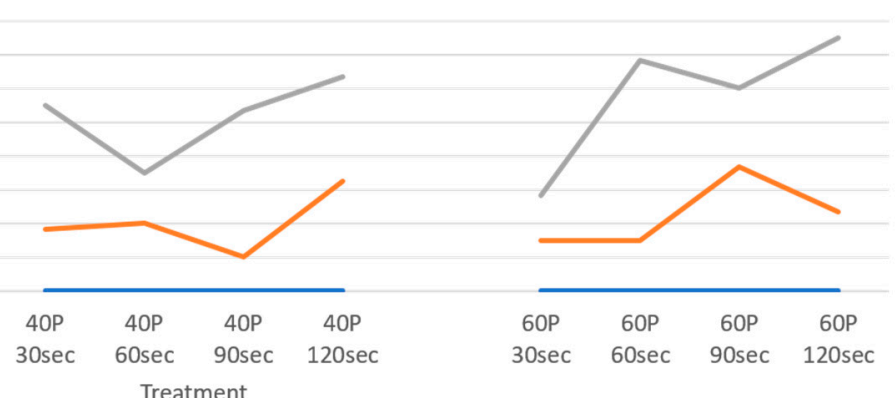

—Canady Helios Cold Plasma Scalpel —Canady Plasma Scalpel Hybrid Mode —Canady Plasma Scalpel APC Mode

Figure 10. Depth of injury caused by each scalpel was measured post-treatment. Treatment conditions were $1 \mathrm{~L} / \mathrm{min}(\mathbf{A}-\mathbf{C}), 3 \mathrm{~L} / \mathrm{min}(\mathbf{D}-\mathbf{F})$, and $5 \mathrm{~L} / \mathrm{min}(\mathbf{G}-\mathbf{I})$ flow rates with different powers and durations.

\section{Discussion}

This study explored the thermal injury between three plasma electrosurgical systems. Due to the differences in the operation and function of each electrosurgical system, a vast amount of data on inflicted tissue damage was collected. Severity of thermal injury, length of each plasma beam, depth of injury, and eschar (lateral spread) was compared between the Canady Plasma ${ }^{\circledR}$ Scalpels.

The Canady Helios Cold Plasma ${ }^{\circledR}$ Scalpel can generate CAP irrespective of the distance between plasma source and target tissue. For comparison purposes, plasma length was sustained at $2 \mathrm{~cm}$ which was typically longer than that of Hybrid and APC modes. It was observed that the CAP jet only attained tissue contact under higher flow rate conditions $(3.0$ and $5.0 \mathrm{~L} / \mathrm{min})$. Despite direct interaction between CAP and tissue in result of longer plasma columns, the eschar diameter and depth of injury remained relatively close to zero and tissue temperature change was minimal for all treatment conditions. The larger distance between the scalpel and the treatment area lessened the direct interaction of plasma with tissue and minimized collateral damage. It was demonstrated that 
the Canady Helios Cold Plasma ${ }^{\circledR}$ Scalpel induced no thermal damage to the normal liver tissue for all conditions tested.

The Canady Plasma ${ }^{\circledR}$ Scalpel in Hybrid and APC modes both depend on the ionization of argon gas but differ in the mechanism by which each plasma is produced. While Hybrid mode is associated with intermittent interruptions in a continuous low voltage waveform with high duty cycle, APC mode relies on spikes in a high voltage waveform with low duty cycle for coagulation. Since direct contact of scalpel and tissue is required for simultaneous cut and coagulation, plasma length could not be measured for Hybrid mode. In contrast, plasma length for APC mode was measurable as APC initiation is contact independent, like CAP mode.

Regarding tissue temperature changes, voltage is positively correlated to temperature. The comparatively lower tissue heating by Hybrid mode is a result of low voltage. Considering physical injury measurements alongside temperature measurements, Hybrid mode can potentially produce incisions and coagulation without causing excessive thermal damages.

The high voltage necessary for APC resulted in notably high temperatures which are ideal for sealing off blood vessels and ultimately preventing blood loss. Since tissue heating consequentially leads to thermal damage, APC produced a greater area of injury compared to Hybrid and CAP modes.

In general, adjustments to CAP parameters had little effect on plasma length, temperature, and injury measurements. For Hybrid mode, plasma length, temperature, and depth of injury also remained unaffected with an increase of plasma dosage. However, it was observed that injury width increased with power. For APC mode, power contributed to plasma length, injury diameter, and injury depth while higher flow rates and longer treatment times induced greater tissue heating.

\section{Conclusions}

Plasma length, temperature change, injury depth, and injury diameter were measured as results of the treatment with the Canady Plasma ${ }^{\circledR}$ Scalpels on different operational settings. Thermal damages varied amongst the three modes. Hybrid mode was found to produce moderate damages while APC mode demonstrated more severe tissue damages. The authors report a new Cold Plasma Jet System caused no thermal or structural damages to normal liver tissue. These findings were significantly less than that of Hybrid and APC modes. With the ability to treat non-cancerous tissue below the threshold of thermal damages, the Canady Helios Cold Plasma ${ }^{\circledR}$ Scalpel has immense potential in cancer therapy.

Supplementary Materials: Data for plasma length, tissue temperature change, eschar width, and depth of injury for all treatment conditions has been included as supplementary materials. Supplementary Materials are available online at http:/ / www.mdpi.com/2571-6182/1/1/17/s1.

Author Contributions: Conceptualization, J.C. and M.K.; Data curation, S.J., T.N. and J.C.; Formal analysis, L.L., S.J., A.S., T.Z., S.W., T.N. and J.C.; Methodology, J.C.; Project administration, J.C.; Resources, J.C. and T.Z.; Writing—original draft, L.L.; Writing—review \& editing, L.L., J.C., T.Z., W.R., X.C., M.K.

Funding: This research was funded by the US Medical Innovations.

Acknowledgments: The authors would like to thank the engineer team at Plasma Medicine Life Sciences for technical support of the plasma unit.

Conflicts of Interest: The authors declare no conflict of interest.

\section{References}

1. D'Arsonval, A. Action physiologique des courants alternatis a grande frequence. Arch. Physiol. Porm. Pathol. 1893, 5, 780-790.

2. Bovie, W.T. Electrosurgical Apparatus. U.S. Patent 1,813,902, 14 July 1931.

3. Cushing, H.; Bovie, W.T. Electrosurgery as an aid to the removal of intracranial tumors. In Surgery, Gynecology and Obstetrics; Surgical Publishing Company: Chicago, IL, USA, 1928.

4. Morrison, C.F., Jr. Electrosurgical Method and Apparatus for Initiating an Electrical Discharge in an Inert Gas Flow. U.S. Patent 40,404,426, 9 August 1977. 
5. Canady, J. Surgical Coagulation Device. U.S. Patent 5,207,675, 4 May 1993.

6. Canady, J.; Vieira, E.; Vieira, N.; Wiley, K. System and Method for Electrosurgical Conductive Gas Cutting for Improving Eschar, Sealing Vessels and Tissues. U.S. Patent 2013/0296846, 2 November 2010.

7. Gjika, E.; Pekker, M.; Shashurin, A.; Shneider, M.; Zhuang, T.; Canady, J.; Keidar, M. The cutting mechanism of the electrosurgical scalpel. J. Phys. D Appl. Phys. 2017, 50, 025401. [CrossRef]

8. Gjika, E.; Scott, D.; Shashurin, A.; Zhuang, T.; Canady, J.; Keidar, M. Plasma-tissue interactions in argon plasma coagulation: Effects of power and tissue resistance. Plasma Med. 2016, 6, 125-134. [CrossRef]

9. Canady, J.; Shashurin, A.; Wiley, K.; Fisch, N.J.; Keidar, M. Characterization of plasma parameters and tissue injury produced by plasma electrosurgical systems. Plasma Med. 2013, 3, 279-289. [CrossRef]

10. Canady, J.; Shashurin, A.; Keidar, M.; Zhuang, T. Integrated Cold Plasma and High Frequency Plasma Electrosurgical System and Method. U.S. Patent 9,999,452, 19 June 2018.

11. Keidar, M.; Shashurin, A.; Volotskova, O.; Ann Stepp, M.; Srinivasan, P.; Sandler, A.; Trink, B. Cold atmospheric plasma in cancer therapy. Phys. Plasmas 2013, 20, 057101. [CrossRef]

12. Guerrero-Preston, R.; Ogawa, T.; Uemura, M.; Shumulinsky, G.; Valle, B.L.; Pirini, F.; Ravi, R.; Sidransky, D.; Keidar, M.; Trink, B. Cold atmospheric plasma treatment selectively targets head and neck squamous cell carcinoma cells. Int. J. Mol. Med. 2014, 34, 941-946. [CrossRef] [PubMed]

13. Kim, S.J.; Chung, T.H. Cold atmospheric plasma jet-generated rons and their selective effects on normal and carcinoma cells. Sci. Rep. 2016, 6, 20332. [CrossRef] [PubMed]

14. Keidar, M.; Walk, R.; Shashurin, A.; Srinivasan, P.; Sandler, A.; Dasgupta, S.; Ravi, R.; Guerrero-Preston, R.; Trink, B. Cold plasma selectivity and the possibility of a paradigm shift in cancer therapy. Br. J. Cancer 2011, 105, 1295-1301. [CrossRef] [PubMed]

15. Kumar, N.; Park, J.H.; Jeon, S.N.; Park, B.S.; Choi, E.H.; Attri, P. The action of microsecond-pulsed plasmaactivated media on the inactivation of human lung cancer cells. J. Phys. D App. Phys. 2016, 49, 115401. [CrossRef]

16. Kumar, N.; Attri, P.; Dewilde, S.; Bogaerts, A. Inactivation of human pancreatic ductal adenocarcinoma with atmospheric plasma treated media and water: A comparative study. J. Phys. D App. Phys. 2018, 51, 255401. [CrossRef]

17. Rowe, W.; Cheng, X.; Ly, L.; Zhuang, T.; Basadonna, G.; Trink, B.; Keidar, M.; Canady, J. The Canady Helios Cold Plasma Scalpel Significantly Decreases Viability in Malignant Solid Tumor Cells in a Dose-Dependent Manner. Plasma 2018. accepted. [CrossRef]

18. Cheng, X.; Rowe, W.; Ly, L.; Shashurin, A.; Zhuang, T.; Wigh, S.; Basadonna, G.; Trink, B.; Keidar, M.; Canady, J. Treatment of Triple-negative Breast Cancer Cells with the Canady Cold Plasma Conversion System: Preliminary Results. Plasma 2018. submitted. 